\title{
EKMAN LAYER SCRUBBING AND SHROUD HEAT TRANSFER IN CENTRIFUGAL BUOYANCY-DRIVEN CONVECTION
}

\author{
Feng Gao, John W. Chew \\ Faculty of Engineering and Physical Sciences \\ University of Surrey \\ Guildford, GU2 7XH, United Kingdom \\ Emails: f.gao@surrey.ac.uk, j.chew@surrey.ac.uk
}

\begin{abstract}
This paper presents large-eddy and direct numerical simulations of buoyancy-driven convection in sealed and open rapidly rotating cavities for Rayleigh numbers in the range $10^{7}-10^{9}$, and axial throughflow Reynolds numbers 2000 and 5600. Viscous heating due to the Ekman layer scrubbing effect, which has previously been found responsible for the difference in sealed cavity shroud Nusselt number predictions between a compressible $\mathrm{N}-\mathrm{S}$ solver and an incompressible counterpart using the Boussinesq approximation, is discussed and scaled up to engine conditions. For the open cavity with an axial throughflow, laminar Ekman layer behaviour of the mean flow statistics is confirmed up to the highest condition in this paper. The Buoyancy number Bo is found useful to indicate the influence of an axial throughflow. For the conditions studied the mean velocities are subject to Ra, while the velocity fluctuations are affected by Bo. A correlation, $N u^{\prime}=0.169\left(R a^{\prime}\right)^{0.318}$, obtained with both the sealed and open cavity shroud heat transfer solutions, agrees with that for free gravitational convection between horizontal plates within $16 \%$ for the range of $R a^{\prime}$ considered.
\end{abstract}

\section{NOMENCLATURE}

\section{Roman Symbols}

$a \quad$ Disc bore radius [m].

$b \quad$ Shroud radius [m].

$d \quad$ Axial gap between discs [m].

$d_{h} \quad$ Axial throughflow hydraulic diameter $=2\left(a-r_{s}\right)$.

$l \quad$ Distance to inlet/outlet [m].

$r \quad$ Radius [m]. $r^{*} \quad$ Non-dimensional radius $=(r-a) /(b-a)$.

$r_{m}=0.5(a+b)$.

$r_{s} \quad$ Shaft radius $[\mathrm{m}]$.

$u_{b} \quad$ Axial throughflow bulk velocity $\left[\mathrm{m} \cdot \mathrm{s}^{-1}\right]$.

$u_{\delta} \quad$ Representative resultant instantaneous velocity at the edge of the disc boundary layer $\left[\mathrm{m} \cdot \mathrm{s}^{-1}\right]$.

$v \quad$ Velocity $\left[\mathrm{m} \cdot \mathrm{s}^{-1}\right]$.

$v_{x}^{*} \quad$ Non-dimensional axial velocity $=v_{x} / u_{b}$.

$v_{r}^{*} \quad$ Non-dimensional radial velocity $=v_{r} /(\Omega r)$.

$v_{\theta}^{*} \quad$ Non-dimensional circumferential velocity $=v_{\theta} /(\Omega r)$.

$x^{*} \quad$ Non-dimensional axial coordinate $=x / d$.

Bo $\quad$ Buoyancy number $=R o / \sqrt{\beta \Delta T}$.

$C_{p} \quad$ Specific heat capacity at constant pressure $=1004.15$ $\left[\mathrm{J} \cdot \mathrm{kg}^{-1} \cdot \mathrm{K}^{-1}\right]$.

$D \quad$ Viscous dissipation rate [W].

Ec $\quad$ Eckert number $=\Omega^{2} r_{m}^{2} /\left(C_{p} \Delta T\right)$.

$\mathrm{Gr} \quad$ Grashof number $=R a / P r$.

$N u \quad$ Shroud Nusselt number $=\frac{\dot{Q} \ln (b / a)}{2 \pi d \kappa \Delta T}$.

$N u^{\prime} \quad$ Modified shroud Nusselt number $=\frac{\dot{Q}(d / 2)}{\kappa\left(T_{b}-T_{\text {core }}\right)}$.

Pr Prandtl number $=0.7$.

$R a \quad$ Rotational Rayleigh number $=\frac{\rho^{2} \Omega^{2} r_{m} \beta \Delta T(b-a)^{3}}{\mu^{2}} P r$.

$R a^{\prime} \quad$ Modified Rayleigh number $=\frac{\rho^{2} \Omega^{2} b \beta\left(T_{b}-T_{\text {core }}\right)(d / 2)^{3}}{\mu^{2}} P r$.

$R e_{\delta} \quad$ Ekman layer Reynolds number $=\frac{u_{\delta} \delta}{v}$.

$R e_{\phi} \quad$ Rotational Reynolds number $=\frac{\rho \Omega r_{m}(b-a)}{\mu}$.

$R e_{x} \quad$ Axial throughflow Reynolds number $=\frac{\rho u_{b} d_{h}}{\mu}$.

Ro $\quad$ Rossby number $=u_{b} /(\Omega a)$.

$S_{\text {disc }} \quad$ Disc surface area $=\pi\left(b^{2}-a^{2}\right)$.

$T \quad$ Static temperature $[\mathrm{K}]$. 
$T^{*} \quad$ Non-dimensional temperature $=\left(T-T_{a}\right) / \Delta T$.

$T_{m} \quad=0.5\left(T_{a}+T_{b}\right)$.

\section{Greek Symbols}

$\beta \quad$ Thermal expansion coefficient $=1 / T_{m}$.

$\beta \Delta T$ Buoyancy parameter.

$\delta \quad$ Ekman depth $=\sqrt{v / \Omega}$.

$\Delta T \quad$ Shaft-to-shroud/inlet temperature difference $=T_{b}-T_{a}$.

$\eta \quad$ Ratio of viscous dissipation to shroud heat transfer.

$\eta_{\mathrm{EL}} \quad$ Ratio of viscous dissipation per unit volume of the Ekman layer to shroud heat transfer per unit volume of the cavity.

$\varepsilon \quad$ Viscous dissipation rate per unit volume $\left[\mathrm{W} \cdot \mathrm{m}^{-3}\right]$.

$\kappa \quad$ Thermal conductivity $=\mu C_{p} / P r$.

$\mu \quad$ Dynamic viscosity $\left[\mathrm{kg} \cdot \mathrm{m}^{-1} \cdot \mathrm{s}^{-1}\right]$.

$v \quad$ Kinematic viscosity $=\mu / \rho$.

$\Omega \quad$ Disc angular speed [rad $\cdot \mathrm{s}^{-1}$ ]

$\rho \quad$ Density $\left[\mathrm{kg} \cdot \mathrm{m}^{-3}\right]$.

\section{Subscripts}

a Disc bore.

$b \quad$ Shroud.

$x, r, \theta$ Axial, radial and circumferential directions.

rms Root mean square.

EL Ekman layer.

\section{Other}

$\langle\cdot\rangle \quad$ Time- and circumferentially-averaged variable.

\section{Acronyms}

DNS Direct numerical simulation.

LES Large-eddy simulation.

MUSCL Monotone upwind schemes for conservation laws.

RANS Reynolds-averaged Navier-Stokes.

URANS Unsteady RANS.

\section{INTRODUCTION}

Modern high-bypass-ratio and high-pressure-ratio turbofan engine design presents multiple challenges. To achieve these design targets the size of the engine core has to be reduced. Without corresponding reductions in blade tip-to-casing clearance this will result in a larger blade tip clearance-to-blade span ratio, which will in turn lead to proportionally higher tip clearance losses. The blade tip clearance of the last axial compressor stage at near nominal engine speed is of the same order of magnitude as the thermal and centrifugal growths of the compressor rotor disc [1]. The thermal growth is determined by the thermal stresses on the discs characterised by the disc temperature distribution, which is associated with the flow and heat transfer in the rotating compressor disc cavities. The thermal stresses also need to be limited at a permissible level for acceptable component life.

Figure 1, reproduced from Fitzpatrick [2], gives an illustration of rotating cavities in a gas turbine high pressure compressor. Open rotating cavities are comprised of the shroud, rotor discs and cobs at the disc bores. Gas in the main annulus gains internal energy through the compression process, leading to high gas temperature. This will heat up the shroud and discs. In most modern engines cooling air taken from the low- or intermediatepressure compressor is directed through the disc bore between the shaft and cobs [3], being expected to extract heat from the high-pressure compressor discs. In some applications a small amount of radial inflow from the shroud is shown to reduce the thermal response time [4,5], whilst closed and unvented cavities occur in some other cases [6]. In many cases these cavity flows are buoyancy-dominated. Therefore, achieving a tight blade tip clearance for modern jet engines requires improved knowledge of buoyancy-driven convection and reliable prediction tools.

\subsection{Closed Rotating Cavity}

Many studies have been conducted to understand the flow physics inside rotating cavities. The experimental study of Bohn et al. [6] gives a simplified representation of a real engine compressor disc cavity, which isolates the natural heat convection from the forced convection induced by an axial throughflow. In Bohn et al.'s study air-filled closed cavities were used to investigate the buoyancy-driven flow under the centrifugal force field. The cavities were bounded by an electrically heated shroud, a water-cooled shaft and two discs assumed to be adiabatic. The heat flux through the shaft surface was measured, and correlations between the Nusselt number $(\mathrm{Nu})$ and the rotational Rayleigh number $(R a)$ were derived for the operating range considered [6,7]. It is also reported that changing the ratio between the radii of the shaft and the shroud $(a / b)$ has negligible influence on $N u$. Among the three geometries used in Bohn et al.'s experiment configuration $\mathrm{B}$, in particular, has motivated a number of numerical [8-13] and theoretical [14] studies. This configuration is shown in Fig. 2 (a).

Sun et al. [8] reported agreement with Bohn et al.'s $N u$ measurements for configuration B through compressible implicit large-eddy simulation (LES) studies. They also observed heat convection through "plumes" of hot and cold air between the shroud and the shaft, forming cyclonic and anti-cyclonic vortices. King et al. [9] conducted two-dimensional (2-D) unsteady

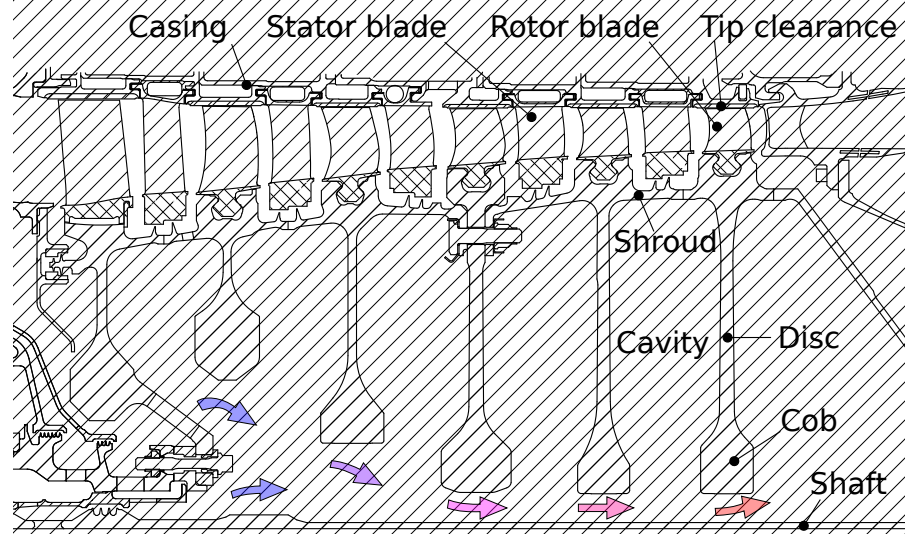

FIGURE 1. ROTOR CAVITIES IN A HIGH PRESSURECOMPRESSOR. REPRODUCED FROM FITZPATRICK [2] 


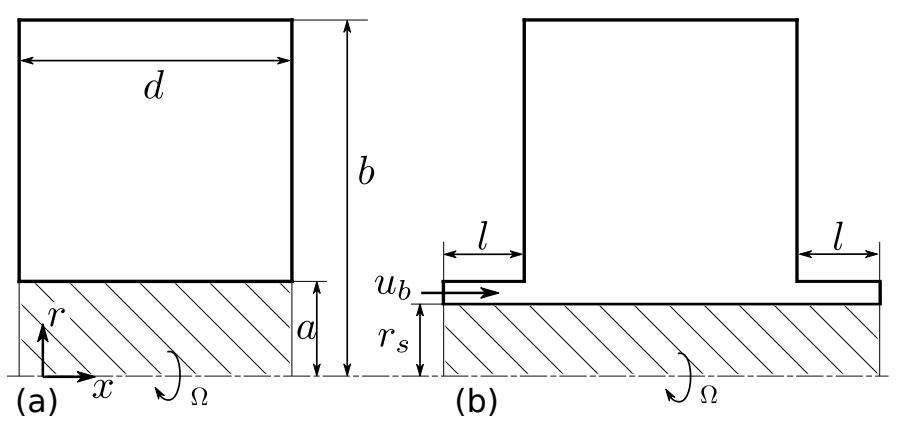

FIGURE 2. (a) BOHN ET AL.'S CONFIGURATION B [6]. (b) EXTENSION TO INCLUDE AN AXIAL THROUGHFLOW [15].

simulations using the Boussinesq approximation, and reported departure of the predicted $N u$ from Bohn et al.'s measurement $\left(N u=0.317 R a^{0.211}\right)$ but agreement with the $N u \propto R a^{1 / 3}$ scaling for gravitational convection between horizontal plates [16]. Pitz et al. [10] reported three-dimensional (3-D) incompressible direct numerical simulation (DNS) with the Boussinesq approximation reproducing $N u \propto R a^{1 / 3}$ scaling. Later Pitz et al. [11] carried out implicit LES studies reporting kinetic and thermal boundary layer statistics of buoyancy flow in a rotating cavity, showing evidence of unsteady laminar Ekman layer behaviour of the disc kinetic boundary layer.

The Boussinesq approximation is well accepted for studying heat convection in geophysics, but is not validated for centrifugal convection, which involves Coriolis forces and a stronger centrifugal force. Motivated by this and the mixed $N u-R a$ scaling found in different studies, the present authors recently compared an incompressible code using the Boussinesq approximation to a fully compressible solver for both 2-D [12] and 3-D [13] models of Bohn et al.'s configuration B. Different degrees of agreement in shroud $\mathrm{Nu}$ between the two solvers were obtained for the 2-D and 3-D configurations, as plotted in Fig. 3. The larger differences between the CFD models for the 3-D configuration at high $R a$ (and higher Eckert number $E c$ ) condition are believed to be caused by the viscous heating due to Ekman layer scrubbing. Despite the differences in $N u$ some general agreement between the two solvers has been confirmed for the $R a$ range $\left(R a \in\left[10^{7}, 10^{9}\right]\right)$ studied. For the 3-D configuration $N u \propto R a^{0.286}$ scaling is obtained for $10^{7} \leq R a \leq 10^{9}$ with the fully compressible solver, which agrees with the $\mathrm{Ra}^{2 / 7}$ scaling for gravitational convection at similar Rayleigh and Prandtl numbers [17].

\subsection{Open Rotating Cavity With Axial Throughflow}

Most engine applications involve open rotating cavities with an axial throughflow. Idealised single rotating cavities with an axial throughflow of air have been experimentally studied by Farthing et al. [18, 19] and Bohn et al. [20]. Engine representative geometries have been studied by Long and co-workers [21-23], Atkins and Kanjirakkad [24], and Puttock-Brown and co-workers [25, 26].

Flow visualisation has revealed the existence of large scale convective flow structures in open rotating cavities with an axial

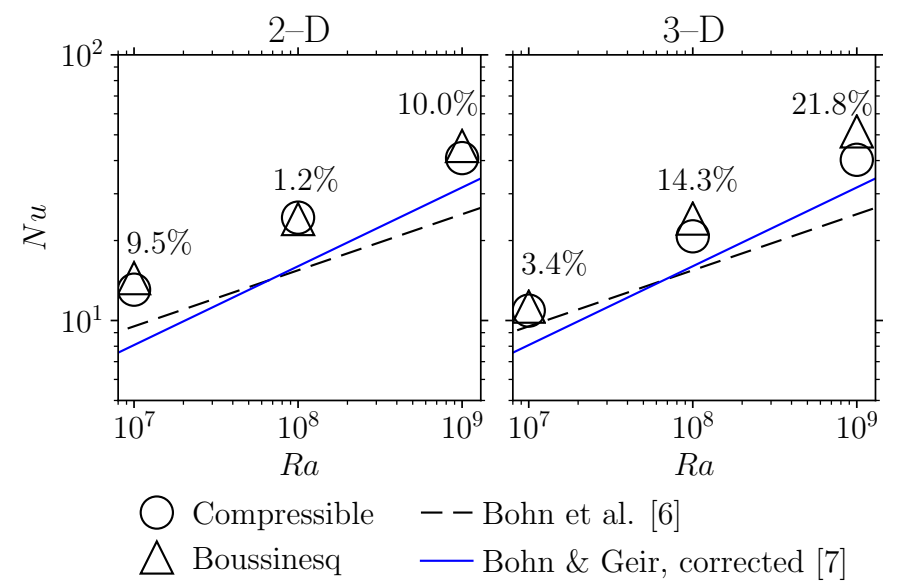

FIGURE 3. SHROUD $N u$ VERSUS Ra FOR THE 2-D [12] AND 3-D [13] CONFIGURATIONS. THE PERCENTAGES CORRESPOND TO THE RELATIVE DIFFERENCES BETWEEN THE COMPRESSIBLE FLOW AND BOUSSINESQ MODELS.

throughflow [18, 20]. Long et al. [23] carried out velocity measurements, using laser Doppler anemometry, in a multiple rotating cavity rig for two different gap sizes between the shaft and the disc bores. At lower radius locations flow rotates faster than the discs for the wide gap configuration, whereas flow rotation slower than disc speed is observed for the narrow gap configuration. In both configurations near solid-body rotation of the flow was measured in the outer region of the cavity. The spectral analysis of the velocity signal confirmed the existence of large scale convective flow structures.

Heat transfer measurements have been performed by Long and co-workers [21, 22], and Puttock-Brown et al. [25]. Most results show that the shroud heat transfer is dependent on the Grashof number $G r$ (or Rayleigh number $R a=\operatorname{Pr} G r$ ) and is insensitive to the axial throughflow Reynolds number $\left(R e_{x}\right)$. There is some evidence that the shroud $N u$ can be approximately correlated using the empirical correlation for Rayleigh-Bénard convection between horizontal plates under gravity [27] by replacing the gravitational acceleration with its centripetal counterpart. This indicates that the cavity flow near the shroud is dominated by free convection. Long and Childs [22] suggested use of the cavity temperature instead of the inlet temperature in this correlation to achieve better agreement. However, in their wide gap $\left(d_{h}\right)$ configuration Long and Childs [22] measured stronger shroud heat transfer, which was attributed to stronger influence from the axial throughflow. For the two axial gap configurations $(d / b)$ studied by Farthing et al. [19] little difference in measured $\mathrm{Nu}$ was observed. In Atkins and Kanjirakkad's [24] experiment, without heat transfer measurement, the influence of different dimensionless parameters on the disc temperature profiles was investigated.

A number of numerical studies have been motivated by the experimental work mentioned above. Bohn et al. [28] simulated their rig [20] with a laminar, compressible, time-dependent flow 
solver, confirming the experimentally observed flow structures rotating slower than the discs. Sun et al. [29] reported LES and URANS calculations of Long et al.'s [21] experiment, with LES showing better agreement with the measurements (within 25\%) than URANS, in terms of the shroud $N u$. Tian et al. [30] reported adequate prediction results of Farthing et al.'s [19] experiment, through steady state simulations. Tan et al. [31,32] reported satisfactory agreement of simulations with Bohn et al.'s [20] measurements using RANS, URANS and LES methods.

Recently, Pitz et al. [15] reported LES calculations extending their sealed cavity study $[6,11]$ to include an axial throughflow. Results were presented for a fixed $R a$ at two different laminar axial throughflow Reynolds numbers $R e_{x}$, and the shroud $N u$ was found to be independent of $R e_{x}$. The cavity core flow was shown to be turbulent, while the flow near the discs showed unsteady laminar Ekman layer behaviour.

In summary, in a sealed rotating cavity flow is dominated by centrifugal free convection, in the presence of an axial throughflow free convection dominates the radially outer part of the cavity and forced convection occurs at the disc bore region. Experimental studies present plausible descriptions of the flow mechanisms but are restricted by limited instrumentation access. LES shows convincing capability to give accurate predictions of the buoyancy-induced flow. The Boussinesq approximation shows good qualitative agreement with the fully compressible equations, but quantitative agreement is subject to conditions studied. The objectives of this paper are to further investigate the Ekman scrubbing effect identified in Ref. [13] and to present further LES studies for a configuration with axial throughflow using a compressible flow solver. Heat transfer results for the sealed and open cavities are compared.

The paper is organised as follows: The configurations studied are described in Section 2. The mathematical modelling is presented in Section 3. Sections 4 and 5 focus on the results and discussion for the sealed cavity and the cavity with an axial through flow, respectively. The main conclusions are summarised in Section 6.

\section{DESCRIPTION OF CONFIGURATIONS STUDIED}

As discussed by Bohn et al. [6] and Farthing et al. [19] the ratio of radii between the shaft/bore and the shroud $(a / b)$ and axial gap between discs $(d / b)$ have relatively little influence on shroud $\mathrm{Nu}$. Hence the geometries of the sealed rotating cavity and the open cavity with axial throughflow are fixed in the present paper. The sealed cavity uses configuration B from Bohn et al. [6] and the open cavity takes the extended configuration by Pitz et al. [15]. These are shown schematically in Fig. 2. The geometric parameters are given in Tab. 1.

In these configurations the rotational Rayleigh number $(R a)$, the rotational Reynolds number $\left(R e_{\phi}\right)$ and the axial throughflow Reynolds number $\left(R e_{x}\right)$ are defined in Eqs. (1)-(3),
TABLE 1. GEOMETRIC PARAMETERS.

\begin{tabular}{cll}
\hline Symbol & Description & Value \\
\hline$a$ & Disc bore radius & $0.125 \mathrm{~m}$ \\
$b$ & Shroud radius & $0.24 \mathrm{~m}$ \\
$d$ & Axial gap between discs & $0.12 \mathrm{~m}$ \\
$r_{s}$ & Shaft radius & $0.115 \mathrm{~m}$ \\
$l$ & Distance to inlet/outlet & $1.5 d$ \\
\hline
\end{tabular}

TABLE 2. OPERATING CONDITIONS.

\begin{tabular}{cllllll}
\hline & \multicolumn{3}{c}{ Sealed cavity } & \multicolumn{3}{c}{ Open cavity } \\
\hline$R a$ & $10^{7}$ & $10^{8}$ & $10^{9}$ & $10^{8}$ & $10^{9}$ & $10^{9}$ \\
$10^{-5} R e_{\phi}$ & 0.114 & 0.412 & 1.48 & 0.412 & 1.48 & 1.48 \\
$\beta \Delta T$ & 0.174 & 0.134 & 0.103 & 0.134 & 0.103 & 0.103 \\
$10^{4} E c$ & 0.382 & 6.60 & 113 & 6.60 & 113 & 113 \\
$R e_{x}$ & - & - & - & 2114 & 1927 & 5674 \\
$R o$ & - & - & - & 0.431 & 0.109 & 0.321 \\
$B o$ & - & - & - & 1.18 & 0.340 & 1.00 \\
\hline
\end{tabular}

$$
\begin{aligned}
R a & =\frac{\rho^{2} \Omega^{2} r_{m} \beta \Delta T(b-a)^{3}}{\mu^{2}} P r \\
R e_{\phi} & =\frac{\rho \Omega r_{m}(b-a)}{\mu} \\
R e_{x} & =\frac{\rho u_{b} d_{h}}{\mu}
\end{aligned}
$$

where $r_{m}=\frac{1}{2}(a+b), \beta \Delta T, u_{b}, d_{h}$ are the arithmetic mean of the shaft and shroud radii, the buoyancy parameter, the axial throughflow bulk velocity and the hydraulic diameter of the axial throughflow inlet, respectively.

To define the rig operating conditions Bohn et al. [6] described a correlation between $R a$ and $R e_{\phi}$,

$$
R e_{\phi}=1.441 R a^{0.557}
$$

Therefore, the six conditions at three $R a$ values considered in this paper are chosen to be consistent with this equation. These are listed in Tab. 2 along with other dimensionless parameters. For all conditions considered in this paper the discs are assumed to be insulated. For the open cavity conditions two different axial throughflow Reynolds numbers-2000 and 5600-were targeted. The $a$ posteriori calculated values are given in the table. These $R e_{x}$ represent laminar and turbulent flow conditions. 


\section{MATHEMATICAL MODELLING}

Two different Navier-Stokes (N-S) equation solvers are used in this paper. One is an industrial code, Hydra [33], developed by Rolls-Royce plc and its university partners. The second is an open source solver, Semtex [34]. A brief description of these two solvers are given below, and readers are referred to Ref. [13] for further information.

\subsection{Hydra}

Hydra solves the compressible N-S equations in finite volume form (Eq. (5)) for unstructured node-vertex meshes in a Cartesian coordinate system. The simulations are performed in a rotating frame of reference, Coriolis and centrifugal forces are included through source terms as in Eq. (6). The centrifugal force term vanishes in the energy equation source term since the convection of rothalpy is considered. A modified Roe scheme based MUSCL scheme is used for spatial discretisation, and second-order accuracy is ensured by linear flux reconstruction techniques [33]. The temporal interpolation employs an explicit three-step Runge-Kutta scheme. The standard Smagorinsky model along with a van Driest damping function is used to simulate the subgrid-scale flow behaviour for LES. The dynamic viscosity is variable following Sutherland's law and other fluid properties are fixed, modelling air as a perfect gas.

$$
\frac{\partial}{\partial t} \int_{V} \vec{W} d V+\oint_{\partial V}\left(\vec{F}_{c}-\vec{F}_{v}\right) d S=\int_{V} \vec{Q} d V
$$

where $\vec{W}$ is the vector of conservative variables, $\vec{F}_{c}$ and $\vec{F}_{v}$ are convective and viscous fluxes, and $\vec{Q}$ is the vector of source terms. $V$ represents to the volume of a grid cell.

$$
\vec{Q}=\left[\begin{array}{c}
0 \\
0 \\
\rho \Omega^{2} y+2 \rho \Omega w \\
\rho \Omega^{2} z-2 \rho \Omega v \\
0
\end{array}\right]
$$

\subsection{Semtex}

Semtex is an incompressible N-S equation solver using a spectral element-Fourier method. The finite difference form of the equation, given in Eq. (7), is solved in a cylindrical coordinate system. The density variation in the centrifugal term is modelled with the Boussinesq approximation $\rho^{\prime}=\rho-\langle\rho\rangle=-\langle\rho\rangle \beta \Delta T$, which is expected to be valid for $\beta \Delta T \lesssim 0.2$. The corresponding source terms are given in Eq. (8). A Poisson equation is solved for pressure, and a temperature transport equation is used for the internal energy. Spectral accuracy is guaranteed using high-order polynomials in each finite element on the meridional plane. Fourier expansions are employed along the homogeneous direction. The convective terms are solved in a skew-symmetric form to avoid aliasing. Time discretisation uses a semi-implicit stiffly stable scheme. No turbulence modelling is used as DNS is performed. All the flow properties are fixed.

$$
\frac{\partial \vec{W}}{\partial t}+\vec{\nabla} \cdot\left(\overline{\bar{F}}_{c}-\overline{\bar{F}}_{v}\right)=\vec{Q}
$$

with $\vec{W}, \overline{\bar{F}}_{c}$ and $\overline{\bar{F}}_{v}$ denoting the primitive variable vector, and tensors of convective and viscous terms.

$$
\vec{Q}=\left[\begin{array}{c}
0 \\
-\beta\left(T-T_{0}\right) \Omega^{2} r-2 \Omega v_{\theta} \\
-2 \Omega v_{r} \\
0
\end{array}\right]
$$

\subsection{Numerical Implementation}

A grid independence study for the sealed cavity configuration was reported in Ref. [13], and finer grids are used for the conditions with axial throughflow. The fine mesh information is given in Tab. 3.

For the sealed cavity adiabatic boundary conditions are adopted on the discs, while isothermal conditions are applied to the shroud and shaft with the shroud temperature $\Delta T$ higher than the shaft temperature.

For the open cavity the shroud is set as isothermal wall, $\Delta T$ above the air inlet temperature, and the other walls are considered adiabatic. A non-reflective static pressure condition is adopted at the outlet. Fixed total pressure is specified at the inlet, which was adjusted to approach the target $R e_{x}$ with an initial RANS solution. The inflow direction is perpendicular to the inlet plane in the rotating frame of reference. The shaft rotates at the same angular speed as the discs.

It should be noted that no perturbations were injected at the inlet for the high $\operatorname{Re}_{x}$ axial throughflow condition. A fully developed turbulent flow at $R e_{x}=5600$ between concentric periodic pipes with radii $r_{s}$ and $a$ was simulated, in order to compare with the unperturbed axial throughflow. The boundary layer profiles are shown in Fig. 4, from the axial throughflow inlet until very close to the upstream disc. The development of mean axial velocity profiles is shown in the left subplot. The boundary layer thickens on both sides as it flows downstream. The velocity fluctuations are plotted in the right sub-figure. Though initial perturbation is not prescribed, the turbulence level intensifies as it develops downstream. The inner region shows weaker turbulence level compared to the periodic flow, while the upper side shows good agreement with the periodic flow data just before entering the cavity. This indicates that the inlet condition for the turbulent axial throughflow is reasonable.

Both Hydra and Semtex have been tested for canonical flows such as periodic pipe flows and rotor stator cavity flows [10,33,35], where Hydra predicted friction velocity agrees with the reference DNS data [36] within 3\%. In addition, the turbulent kinetic energy budget, which is an important factor for the fidelity of Navier-Stokes solvers, has been verified [13, 35], with less than $80 \%$ of the total dissipation represented by the 
TABLE 3. NUMERICAL PARAMETERS. $\Delta_{w}$ : NEAR WALL GRID HEIGHT. $N_{\theta}$ : GRID NODE NUMBER IN THE CIRCUMFERENTIAL DIRECTION. $N_{\text {tot }}$ : TOTAL GRID NODE NUMBER. $\Delta t$ : TIME STEP IN ROTOR REVOLUTION. M: MILLION.

\begin{tabular}{|c|c|c|c|c|c|c|c|}
\hline & & \multicolumn{3}{|c|}{ Sealed cavity } & \multicolumn{3}{|c|}{ Open cavity } \\
\hline & $R a$ & $10^{7}$ & $10^{8}$ & $10^{9}$ & $10^{8}$ & $10^{9}$ & $10^{9}$ \\
\hline & $R e_{x}$ & - & - & - & 2114 & 1927 & 5674 \\
\hline \multirow{4}{*}{$\underset{5}{5}$} & $\Delta_{w}[\mathrm{~mm}]$ & 0.2 & 0.1 & 0.05 & 0.08 & 0.05 & 0.05 \\
\hline & $N_{\theta}$ & 200 & 600 & 600 & 600 & 800 & 800 \\
\hline & $N_{\text {tot }}$ & $\sim 0.7 \mathrm{M}$ & $\sim 3.4 \mathrm{M}$ & $\sim 4.3 \mathrm{M}$ & $\sim 8.6 \mathrm{M}$ & $\sim 13 \mathrm{M}$ & $\sim 13 \mathrm{M}$ \\
\hline & $\Delta t[\mathrm{rev}]$. & $7.59 \times 10^{-7}$ & $1.37 \times 10^{-6}$ & $2.47 \times 10^{-6}$ & $1.14 \times 10^{-6}$ & $2.30 \times 10^{-6}$ & $2.30 \times 10^{-6}$ \\
\hline \multirow{4}{*}{ 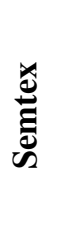 } & $\Delta_{w}[\mathrm{~mm}]$ & 0.077 & 0.077 & 0.04 & - & - & - \\
\hline & $N_{\theta}$ & 384 & 384 & 640 & - & - & - \\
\hline & $N_{\text {tot }}$ & $\sim 9.1 \mathrm{M}$ & $\sim 9.1 \mathrm{M}$ & $\sim 52.3 \mathrm{M}$ & - & - & - \\
\hline & $\Delta t$ [rev.] & $2.53 \times 10^{-4}$ & $2.28 \times 10^{-4}$ & $6.58 \times 10^{-5}$ & - & - & - \\
\hline
\end{tabular}

subgrid-scale and numerical dissipation. These, along with the axial through flow validation in the previous paragraph, indicate a high level of confidence in the present simulations. The sealed cavity calculations have been validated against experimental measurement of shroud heat transfer [13], showing agreement between the experimental correlation and Hydra solutions within $21 \%$. Other workers have noted significant uncertainty in the heat transfer measurements [7] that could account for some or all of this difference.
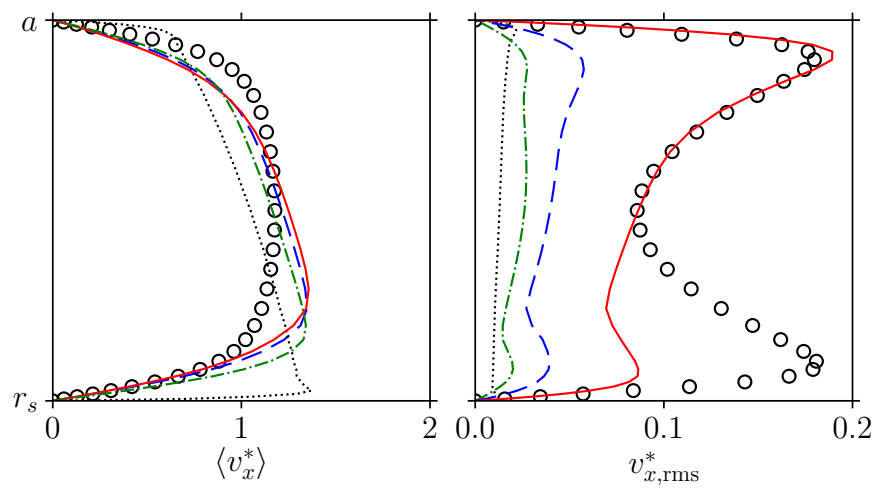

$\begin{array}{cl}\circ \quad \text { Periodic } & \cdots \cdots \cdots \cdot x=-1.50 d \quad--\cdot x=-1.00 d \\ --x=-0.50 d & -x=-0.14 d\end{array}$

FIGURE 4. AXIAL THROUGHFLOW BOUNDARY LAYER PROFILES FOR $R e_{x}=5674$. THE VALUE OF $x$ INDICATES THE RELATIVE AXIAL POSITION TO THE UPSTREAM DISC. $\left\langle v_{x}^{*}\right\rangle=\left\langle v_{x}\right\rangle / u_{b}$ IS THE NORMALISED MEAN AXIAL VELOCITY. $v_{x, \mathrm{rms}}^{*}$ DENOTES THE ROOT MEAN SQUARE OF THE VELOCITY FLUCTUATIONS.

\section{SEALED ROTATING CAVITY}

Some results from the sealed cavity simulations are presented in Ref. [13]. This section gives further explanation and analysis of the Ekman layer scrubbing effect.

\subsection{Static Temperature}

Time- and circumferentially-averaged static temperature contours are shown in Fig. 5 on the $x-r$ plane for Hydra solutions. The mean temperature is normalised with the shaft-toshroud temperature difference as $\left\langle T^{*}\right\rangle=\left(\langle T\rangle-T_{a}\right) /\left(T_{b}-T_{a}\right)$. Time-averages were taken over at least 50 disc revolutions. A similar level of uniform core temperature is observed in the three plots. The shroud and shaft thermal boundary layers thin as $R a$ increases. For the two lower $R a$ cases there is slight asymmetry about the mid-axial position. This indicates either that an asymmetric mean flow develops in these cases, or that the timescale for flow variation is relatively long. At $R a=10^{9}$, the temperature contours are symmetric and show higher temperature near the discs than at the mid-axial position.
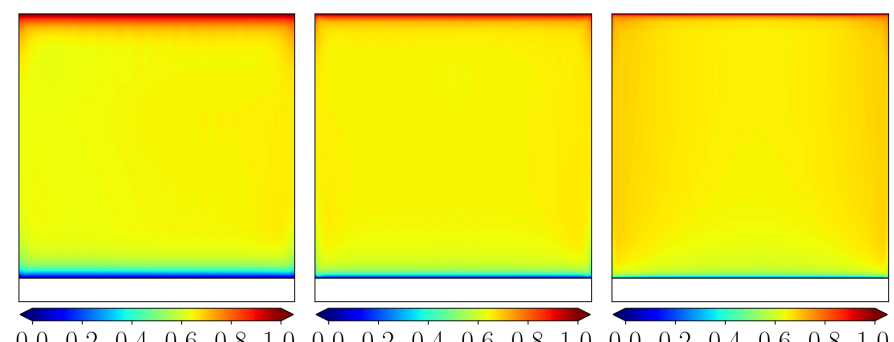
(a) $R a=10^{7}$
(b) $R a=10^{8}$
(c) $R a=10^{9}$

FIGURE 5. CONTOUR PLOTS OF THE TIME- AND CIRCUMFERENTIALLY-AVERAGED TEMPERATURE IN THE SEALED CAVITY. HYDRA SOLUTIONS. 
A further plot in Fig. 6 shows the temperature profiles on the disc and midway between the discs. The disc temperature profiles are further averaged with those from the two discs. Both profiles show uniform temperature away from the cylindrical walls for all the conditions studied. With Hydra results similar temperatures are found in the core and on the discs at lower $R a$ values, whereas for $R a=10^{9}$ disc temperature is slightly higher than that in the core. The plot at $R a=10^{9}$ shows very good agreement between Hydra and Semtex in terms of the core temperature profile. But the shroud $N u$ differs by $21.8 \%$ as shown in Fig. 3. This is attributed to the difference in disc temperature caused by the Ekman layer scrubbing effect as will be discussed in the next subsection. Figure 6 also shows Semtex's disc temperature differing from that at the core for lower $R a$ conditions. Differences between Hydra and Semtex may be associated with the effect of high $\beta \Delta T$, which approaches the Boussinesq approximation validity margin. Both solvers show some evidence of large scale flow structures in the core at the lower $R a$, which is also reported in Ref. [37]. However, as shown in Fig. 3 the integrated value of the shroud $N u$ can be considered acceptable.
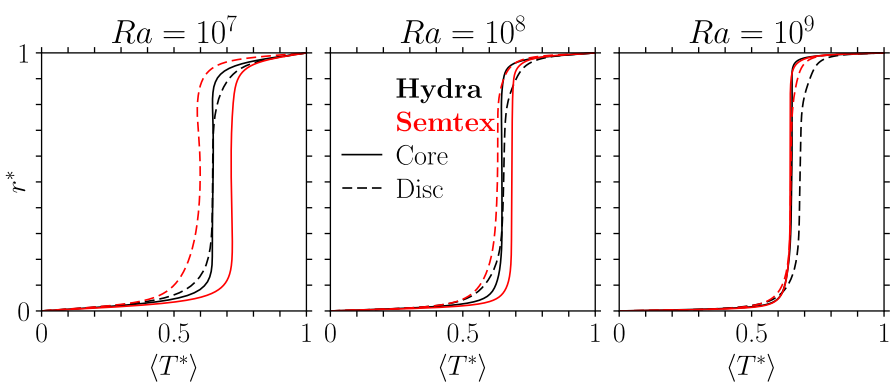

FIGURE 6. TIME- AND CIRCUMFERENTIALLY-AVERAGED RADIAL TEMPERATURE PROFILES FOR THE SEALED CAVITY.

\subsection{Ekman Layer Scrubbing}

As mentioned above the difference between disc and core temperatures in the Hydra solution at $R a=10^{9}$ is associated with the viscous heating effect due to Ekman layer scrubbing, and this is considered mostly responsible for the difference in $\mathrm{Nu}$ between the two solvers.

As shown by Gao et al. [13] the mean velocities in the sealed cavity are statistically close to zero but instantaneous velocities are much stronger. The unsteady velocity component drives unsteady Ekman layers on the discs which convert kinetic energy to internal energy through viscous dissipation. The internal energy that is not convected away (owing to the small mean velocity) accumulates near the discs. This effect will be more significant at higher rotational speeds representative of engine conditions.

Scaling of the Ekman layer scrubbing effect up to higher speed is considered here. For the disc boundary layers considered the rate per unit volume of conversion of kinetic energy to internal energy is

$$
\varepsilon_{\mathrm{EL}}=\mu\left\langle\left(\frac{\partial v_{\theta}}{\partial x}\right)^{2}+\left(\frac{\partial v_{r}}{\partial x}\right)^{2}\right\rangle
$$

Integrating across the boundary layer and the disc surface gives the total energy dissipation from one disc,

$$
D_{\mathrm{EL}}=\int_{S_{\mathrm{disc}}} \int_{0}^{\infty} \varepsilon_{\mathrm{EL}} d x d S .
$$

This can be estimated using the Ekman layer solution, giving

$$
D_{\mathrm{EL}}=S_{\mathrm{disc}} \rho\left\langle u_{\delta}^{2}\right\rangle \sqrt{\Omega v}
$$

where $u_{\delta}$ is the representative resultant instantaneous velocity at the edge of the boundary layer. $S_{\mathrm{disc}}=\pi\left(b^{2}-a^{2}\right)$ is the surface area of one disc.

For comparison, the total heat through the shroud may be written

$$
\dot{Q}=N u \frac{2 \pi d \kappa \Delta T}{\ln (b / a)}
$$

with $\kappa=\mu C_{p} / \operatorname{Pr}$ being the thermal conductivity.

Our previous work [13] shows the root mean square of the velocity fluctuation is of order $0.2 \Omega r \sqrt{\beta \Delta T}$ (this can replace the velocity $u_{\delta}$ in Eq. (11)), and $N u$ is of order $0.11 R a^{2 / 7}$. The ratio of the internal energy generation (in Eq. (11)) from both discs to the shroud total heat transfer (in Eq. (12)) can then be estimated as follows,

$$
\eta=\frac{2 D_{\mathrm{EL}}}{\dot{Q}}=\frac{0.36\left(b^{2}-a^{2}\right) \ln (b / a)}{r_{m}^{3 / 14}(b-a)^{11 / 14} d} \frac{E c(\beta \Delta T)^{5 / 7} \operatorname{Pr}^{5 / 7}}{\operatorname{Re}_{\phi}^{1 / 14}}
$$

where $E c=\left(\Omega^{2} r^{2}\right) /\left(C_{p} \Delta T\right)$ is the Eckert number. Neglecting the geometrical length and constants, Equation (13) gives

$$
\eta \propto \frac{E c(\beta \Delta T)^{5 / 7} \operatorname{Pr}^{5 / 7}}{\operatorname{Re}_{\phi}^{1 / 14}} .
$$

The estimated ratio of the viscous heating due to Ekman layer scrubbing to the shroud heat transfer $\eta$ is plotted in Fig. 7. For Hydra solutions $\eta=2 D_{\mathrm{EL}} / \dot{Q}$ is directly calculated using $D_{\mathrm{EL}}$ from Eq. (10) and the unsteady flow solutions. The theoretical solution of $\eta$ is computed with Eq. (13), for Bohn et al.'s [6] rig conditions. Using the correlation in Eq. (4), which defines the rig operating condition, the non-dimensional variables in Eq. (13) for each $R a$ can be calculated if a rig pressure or rig speed is assumed. For $R a \leq 10^{10}$ the curve given assumes atmospheric pressure (as in the CFD results). For $R a>10^{10}$ the rig is assumed to operate at its maximum speed of $3500 \mathrm{rpm}$. At the maximum $R a$ value of $10^{11}$ the corresponding pressure is close to the maximum experimental value of 4 bar. The top subplot in Fig. 7 shows the ratio of the viscous heating to the total shroud heat transfer. The bottom sub-figure plots the ratio of the viscous heating per unit volume of the Ekman layer to the shroud heat transfer per unit volume of the cavity $\left(\eta_{\mathrm{EL}}=\eta d /\left(2 \delta_{\mathrm{EL}}\right)\right)$.

In the top subplot $\eta$ is $\sim 5.7 \times 10^{-2} \%$ at $R a=10^{9}$. As the instantaneous velocity fluctuates around a near-zero mean value, 

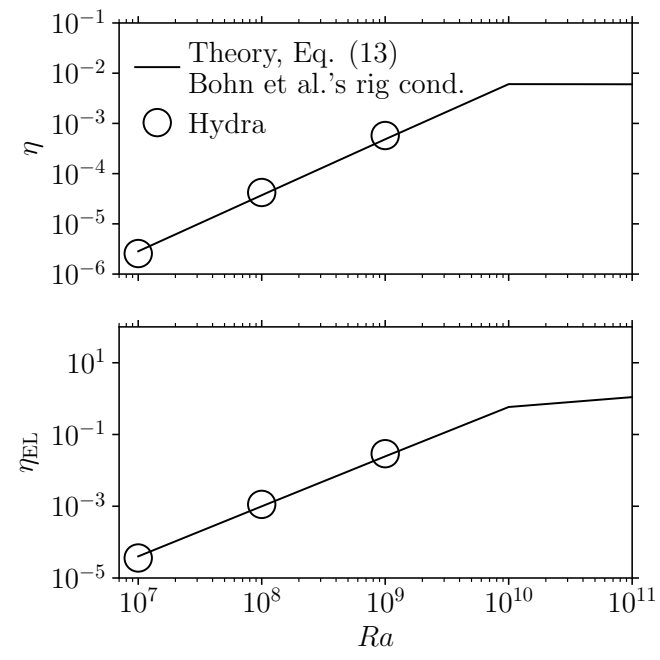

FIGURE 7. TOP: RAITO OF VISCOUS DISSIPATION TO TOTAL HEAT CONVECTION. BOTTOM: RATIO OF VISCOUS DISSIPATION PER UNIT VOLUME OF THE EKMAN LAYER TO HEAT CONVECTION PER UNIT VOLUME OF THE CAVITY.

the viscous heat generated is unlikely to be convected away from the Ekman layer. Therefore, it is also of interest to plot $\eta_{\mathrm{EL}}$. Indeed, $\eta_{\mathrm{EL}}$ goes up to $\sim 2.9 \%$ at $R a=10^{9}$, which is consistent with heating of air in the disc Ekman layer, as shown by the disc temperature in Fig. 6. The temperature difference between the core and the disc shows a similar order of magnitude to $\eta_{\mathrm{EL}}$. As discussed above the internal energy gained through viscous dissipation cannot be convected away due to small convection velocity, and accumulates near the discs.

Excellent agreement between the numerical results and the theoretical solution is achieved in both subplots, indicating good performance of the theoretical correlation in Eq. (13). Note, however, that the scaling constant for $u_{\delta}$ is somewhat arbitrary. Assuming Eq. (13) is valid up to Bohn et al.'s highest speed condition $\left(R a \geq 10^{10}\right)$, viscous heating per unit volume of the Ekman layer and the shroud heat transfer per unit volume of the cavity can have the same order of magnitude. Some coupling of the flow velocities with the Ekman layer scrubbing may occur at high $R a$ conditions, modifying the scaling given above.

Note that the discussion above is based on a laminar Ekman layer assumption, and a discussion on its transition to turbulence is given in this paragraph. As discussed by Greenspan [38] the first instability of a laminar Ekman layer occurs at $R e_{\delta} \approx 56$, where $R e_{\delta}$ is the Ekman layer Reynolds number defined as

$$
\operatorname{Re}_{\delta}=\frac{\left\langle u_{\delta}\right\rangle \delta}{v}=\frac{\left\langle u_{\delta}\right\rangle}{\Omega b \sqrt{\beta \Delta T}} \frac{b \sqrt{\beta \Delta T}}{r_{m}^{1 / 2}(b-a)^{1 / 2}} \operatorname{Re}_{\phi}^{1 / 2},
$$

with $\delta=(v / \Omega)^{0.5}$ being the Ekman depth. However, this critical Reynolds number $R e_{\delta}$ does not necessarily trigger the transition to fully developed turbulence. According to Owen and Pincombe [39] transition may be delayed to $R e_{\delta} \approx 200$. This transitional $R e_{\delta}$ translates to $\left\langle u_{\delta}\right\rangle /(\Omega b \sqrt{\beta \Delta T})=0.35$ at $R a=10^{11}$ (above the highest $R a$ condition considered in Bohn et al.'s experiment). This is higher than the velocity scaling $0.2 \Omega b \sqrt{\beta \Delta T}$ from our previous study [13], and the Ekman layer will still be laminar. Hence, using the laminar Ekman layer assumption here is justified.

Figure 8 maps test conditions for Bohn et al.'s rig B on a graph of Eckert number against $\beta \Delta T$. Other lines on the graph indicate the significance of heating due to Ekman layer scrubbing, transition to turbulent in the Ekman layers, and effects of compressibility. As above, the rig conditions considered here assume an operating pressure of 1 atmosphere (at lower $\mathrm{Ra}$ ) or operation at the maximum rig speed of $3500 \mathrm{rpm}$ (at higher $R a$ ). Assuming the rig pressure never fell below 1 atmosphere this gives the upper limit of the Eckert number for the rig at each value of $\beta \Delta T$. Black solid curves represent constant $\eta$ at $R e_{\phi}=10^{6}$ according to Eq. (13). Blue curves vary $R e_{\phi}$ indicating limited sensitivity to $R e_{\phi}$. The present simulations at $R a=10^{9}$ showed significant effects of Ekman layer scrubbing, and the map indicates that $\eta$ and the scrubbing effect will be stronger at higher $R a$. The vertical dashed lines present the critical $\beta \Delta T$ at given values of $R e_{\phi}$ for which transition to turbulence is indicated by Eq. (14), assuming the same velocity scaling as used in deriving Eq. (13). The maximum value of $R e_{\phi}$ in the experiments is about $2 \times 10^{6}$, so the Ekman layers are expected to remain laminar at these conditions.

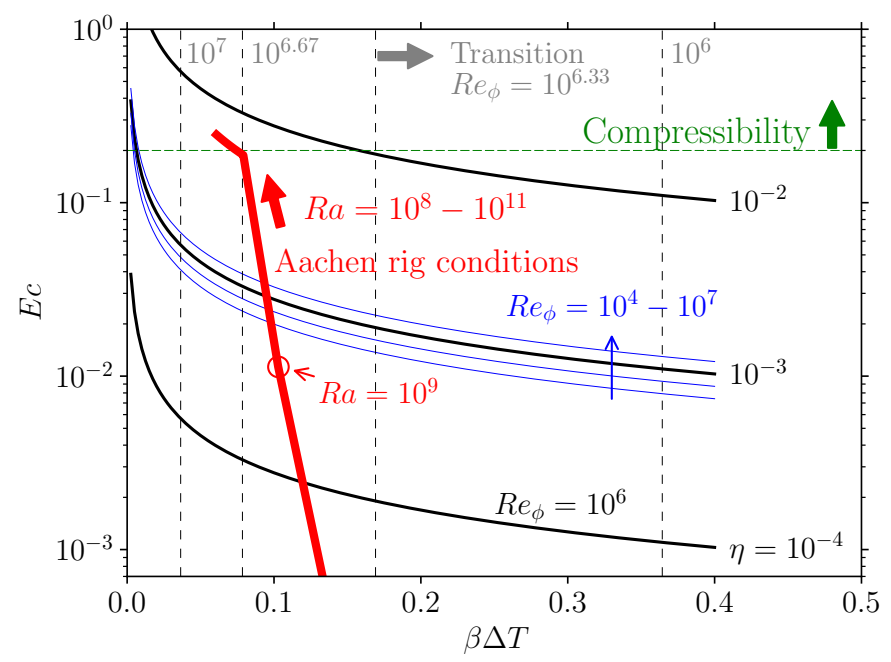

FIGURE 8. MAP OF OPERATING CONDITIONS SHOWING CRITERIA FOR EFFECT OF COMPRESSIBILITY, EKMAN LAYER TRANSITION AND VISCOUS HEATING.

Compressibility effects are expected to be significant at high values of Ec. As discussed in Ref. [40], an isentropic forced vortex in a compressible gas can support a negative density gradient and corresponding positive radial temperature gradient. In such a vortex the rothalpy $\left(=C_{p} T-0.5 \Omega^{2} r^{2}\right.$ in a forced vortex) is constant. At $E c=0.2$, indicated by the horizontal dashed line in Fig. 8, an isentropic forced vortex at disc speed will give a radial temperature difference between inner and outer radii of the present configuration of $12.6 \%$ of the imposed temperature 
difference. For the simulations presented here the compressibility effect is small, but at the highest Rayleigh number tested by Bohn et al. $\left(\sim 5 \times 10^{10}\right)$ compressibility effects are becoming significant.

It should be noted that engine conditions may differ from Bohn et al.'s rig in terms of both geometry and flow conditions, and that wide variation in flow conditions can occur during a flight cycle. Higher speeds and pressures will lead to higher Reynolds, Rayleigh and Eckert numbers. Thus at some engine conditions the Ekman layers are likely to become turbulent and compressibility effects will be significant. However these effects may interact. The radial temperature gradient in the rotating core at high Eckert number will effectively reduce the driving temperature for the convection. This might be accounted for in Fig. 8 by subtracting the temperature difference across the core from the shroud-to-hub temperature difference $\Delta T$, and this reduces the likelihood of turbulent transition. Ekman layer transition is likely to affect the disc heat transfer. The heating due to Ekman layer scrubbing is always relatively small compared to the shroud heat transfer but, as seen in the simulations for adiabatic discs, may affect temperatures in the disc boundary layers and core. In engine applications the significance will depend on the level of disc heat transfer.

\section{ROTATING CAVITY WITH AN AXIAL THROUGH- FLOW}

Figure 9 shows instantaneous temperature contours for the three axial throughflow cases studied on the upstream and downstream discs, and in the core. "Plumes" seen in the sealed cavity [13] are again present driving heat transfer between the hot shroud and the cold axial throughflow. At high buoyancy numbers $(B o=R o / \sqrt{\beta \Delta T})$ the downstream disc surface is colder than the upstream disc and vice versa. Note also that there is a change in lobe number between the high and low Bo conditions. Animations showing evolutions of the core temperature can be watched with the PDF file.

\subsection{Mean Flow Field}

Time- and circumferentially-averaged axial velocity contours, normalised by the inlet bulk velocity $u_{b}$, are shown in Fig. 10. The high $B o$ conditions show a stronger jet through the cavity. The near-shroud area stays relatively quiescent in all three conditions.

As discussed by many researchers, for example in Refs. $[30,41]$, a toroidal vortex is expected with an axial througflow of air. This can be seen through streamline plots in Fig. 11. Also shown on the figure are the mean radial velocity contours. Three main vortices are observed for all three cases. Though the streamlines do not reflect the strength of the vortex, this can be gauged from the radial velocity contours. For the two high $B o$ cases (with similar $B o$ values) a strong vortex is found in the inner part of the cavity. These vortex cores are closer to the downstream disc compared to the low Bo case. The corresponding outer vortex (close to the shroud) also shows core being closer to
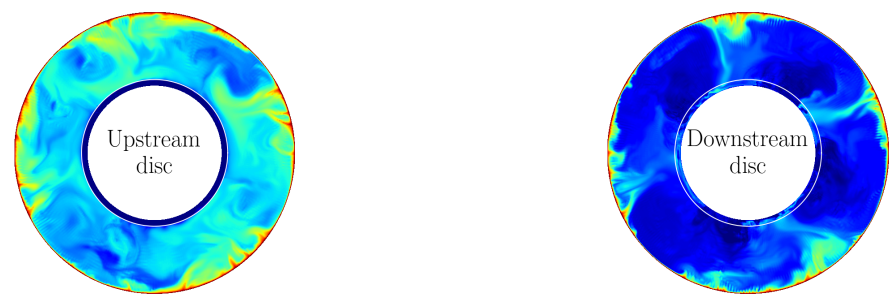

$R a=10^{8}, B o=1.18$
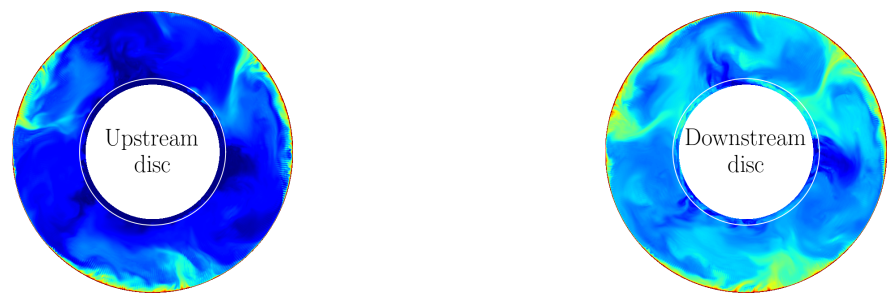

$R a=10^{9}, B o=0.34$
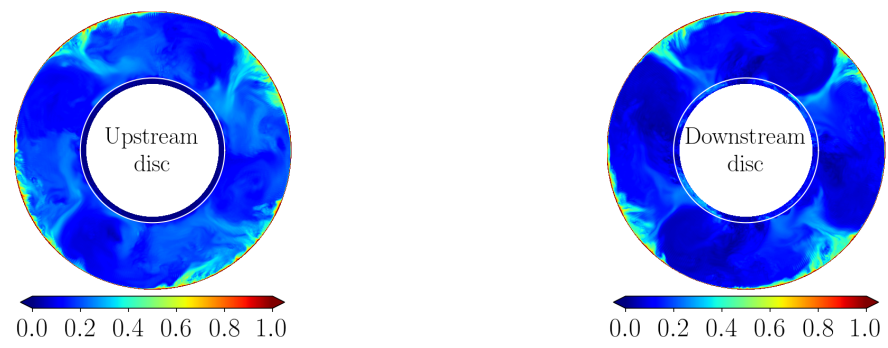

$$
R a=10^{9}, B o=1.0
$$

FIGURE 9. INSTANTANEOUS NORMALISED TEMPERATURE CONTOURS ON THE DISCS AND IN THE CORE. THE WHITE CIRCLE NEAR THE SHAFT INDICATES THE DISC BORE RADIUS. CLICK ON THE MIDDLE COLUMN PLOTS TO WATCH ANIMATIONS.
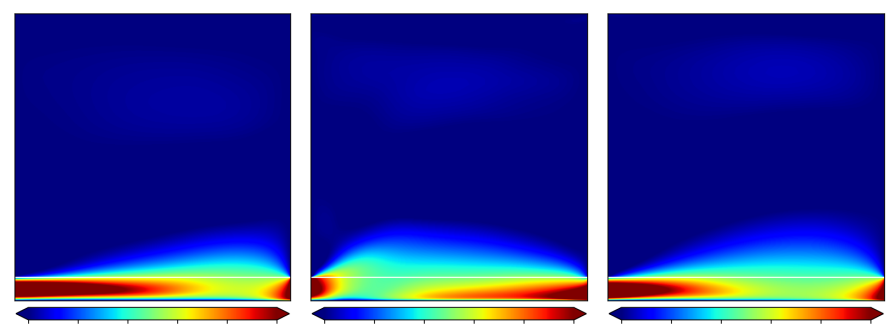

$\begin{array}{lllllll}0.0 & 0.2 & 0.4 & 0.6 & 0.8 & 1.0\end{array}$

(a) $R a=10^{8}, B o=1.18$

(b) $R a=10^{9}, B o=0.34$

(c) $R a=10^{9}, B o=1.0$

FIGURE 10. CONTOUR PLOTS OF THE MEAN AXIAL VELOCITY $\left(\left\langle v_{x}^{*}\right\rangle=\left\langle v_{x}\right\rangle / u_{b}\right)$. THE WHITE STRAIGHT LINE NEAR THE SHAFT INDICATES THE DISC BORE RADIUS.

the downstream disc. In the low Bo case the vortices are weaker, as illustrated by the velocity contour at the edge of the vortices. The vortices at the shroud are the weakest in all three cases.

In terms of disc kinetic boundary layer Pitz et al. [15] reported unsteady Ekman layer behaviour at $R a=10^{8}$ with a laminar axial throughflow. Here further boundary layer statistics at a higher $R a$ value and with both laminar and turbulent axial 

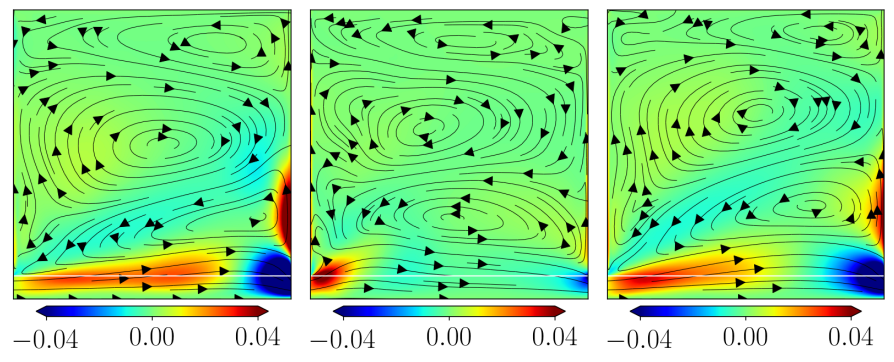

(a) $R a=10^{8}, \mathrm{Bo}=1.18$

(b) $R a=10^{9}, B o=0.34$

(c) $R a=10^{9}, B o=1.0$

FIGURE 11. MEAN FLOW FIELD STREAMLINES WITH MEAN RADIAL VELOCITY CONTOUR $\left(\left\langle v_{r}^{*}\right\rangle=\left\langle v_{r}\right\rangle /(\Omega r)\right)$. THE WHITE STRAIGHT LINE NEAR THE SHAFT CORRESPONDS TO THE DISC BORE RADIUS.

throughflow are presented.

Time- and circumferentially-averaged radial velocity profiles are plotted in Fig. 12. On the upstream disc the boundary layer thickness indicated by radial velocity profiles corresponds approximately to the laminar Ekman layer solution represented by the vertical straight lines. The $R a$ effect is clear as the curves at $R a=10^{9}$ are similar. All three cases show a general decrease of radial velocity magnitude as $r^{*}$ increases. A reversal of $\left\langle v_{r}^{*}\right\rangle$ occurs between $r^{*}=0.7$ and 0.8 at $R a=10^{8}$. Clear effects of the axial throughflow are seen at inner radii near the downstream disc. This influence extends to a radial position between $r^{*}=0.4$ and 0.5 at high $B o$ conditions.

In contrast to the mean radial velocity, its fluctuation shows effects of $B o$ rather than $R a$. This can be seen through the better match between the curves with a similar Bo, as shown in Fig. 13 . Laminar Ekman layer behaviour is also confirmed by the radial velocity fluctuations. The downstream disc regions affected by the axial throughflow jet also show stronger fluctuation than the upstream disc.

In the sealed rotating cavity fluid moves close to solid body rotation with the discs. But the tangential speed of fluid in an open cavity with axial throughflow depends on the Rossby number $R o$ [23]. Figure 14 shows the time- and circumferentially averaged tangential velocity and its fluctuation profiles at the mid-axial position between the discs. Negative tangential velocity $\left\langle v_{\theta}^{*}\right\rangle$ in the rotating frame of reference is obtained in all the cases, indicating that the angular speed of the fluid is slower than the discs. This corresponds to the mean radial outflow in the disc Ekman layer. In the upper part of the cavity $\left(r^{*}>0.4\right)$ an effect of $R a$ is observed. For the velocity fluctuations agreement between the two high $B o$ cases can be observed throughout the radial extent plotted, indicating the $B o$ effect is dominant. This behaviour is consistent with that observed for the radial velocity statistics.

\subsection{Heat Transfer}

Time- and circumferentially-averaged static temperature contours are shown in Fig. 15. At $R a=10^{8}$ the upstream disc temperature is higher than the downstream disc. Increasing $R a$ to $10^{9}$ and keeping the same axial throughflow $R e_{x}$, the upstream disc becomes colder than the downstream disc. This is associated with the reduction in $B o$. Increasing $R e_{x}$ further the temperature field becomes more uniform, as shown in the subplot (c).

Figure 16 compares the mean temperature profiles on the two discs and in the core. The shroud thermal boundary layer is observed through high temperature gradient. Away from the shroud a fairly uniform temperature profile is obtained, as for the sealed cavity. At the upstream disc bore a strong temperature gradient is seen due to the sharp temperature difference between the cavity fluid and the cold axial throughflow. The temperature is more uniform at the downstream disc bore owing to the mixing in the cavity. The upstream disc temperature shows close agreement between the two cases with the same $\mathrm{Ra}$. In contrast, the downstream disc shows closer agreement between the two cases with a similar $B o$, indicating the effect of $B o$ dominates the downstream disc temperature.

The shroud $N u$ distribution along the axial direction is plotted in Fig. 17. Similar $N u$ distributions are found near the upstream disc for the two cases with $R a=10^{9}$, as is consistent with the observation made for velocity and temperature profiles. For the higher $B o$ case the axial throughflow jet is stronger, enhancing the heat transfer near the downstream disc. For a similar $B o$ the $N u$ distribution at $R a=10^{8}$ shows a similar trend, i.e. stronger jet results in higher heat transfer near the downstream disc.

Many studies, as reviewed by Owen and Long [3], have suggested free convection occurring at the shroud . Long and Childs [22] employed Lloyd and Moran's [27] correlation to scale their heat transfer measurements, using a Nusselt number based on the core-to-shroud temperature difference. The modified ${ }^{1}$ Rayleigh number $R a^{\prime}$ and the modified Nusselt number $N u^{\prime}$ are given in Eqs. (15) and (16). The characteristic length scale is defined as the ratio of the area subject to heat transfer to its perimeter [27]. In this paper $d / 2$ is used.

$$
\begin{gathered}
R a^{\prime}=\frac{\rho^{2} \Omega^{2} b \beta\left(T_{b}-T_{\text {core }}\right)(d / 2)^{3}}{\mu^{2}} P r \\
N u^{\prime}=\frac{\dot{Q}(d / 2)}{\kappa\left(T_{b}-T_{\text {core }}\right)}
\end{gathered}
$$

The modified shroud $N u^{\prime}$ from both the sealed and open cavities are plotted in Fig. 18 for Hydra solutions. Two correlations for low $\left(10^{4} \lesssim R a^{\prime} \lesssim 10^{7}\right)$ and high $\left(10^{7} \lesssim R a^{\prime} \lesssim 10^{9}\right)$ Rayleigh numbers [27] are also given in the plot. Good agreement between the LES data and the correlation lines is shown, which confirms free convection near the shroud. For high $R a^{\prime}$ values the LES data scales better with the correlation at the same $R a^{\prime}$ range, i.e. it has a similar scaling exponent. Correlating the LES data, the following correlation function is obtained,

$$
N u^{\prime}=0.169\left(R a^{\prime}\right)^{0.318} .
$$

\footnotetext{
${ }^{1}$ The term "modified" is used to differentiate cases using core and shaft/inlet temperatures.
} 

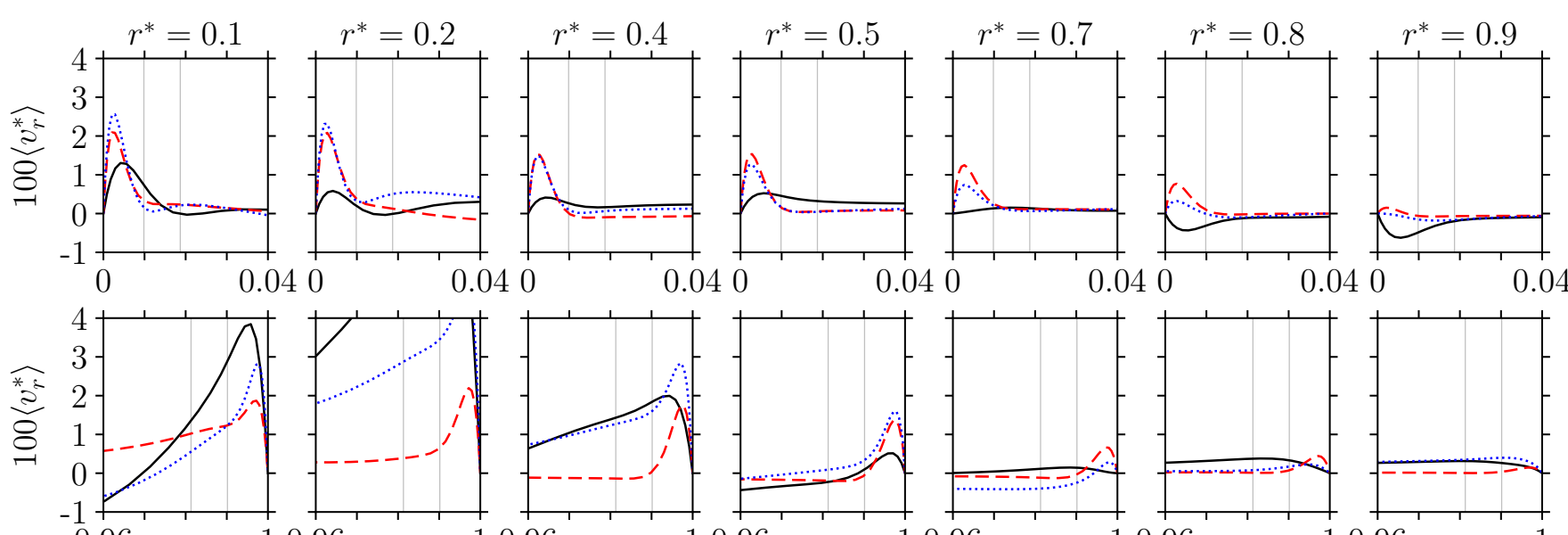

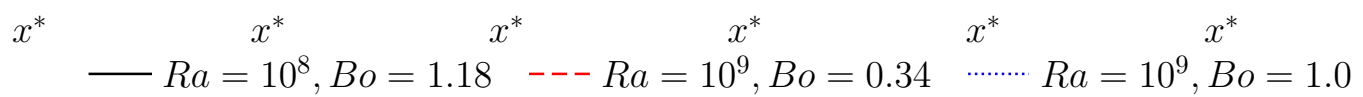

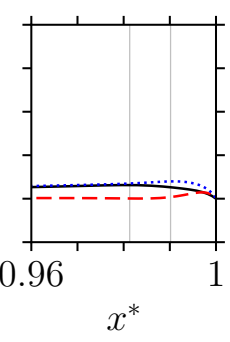

FIGURE 12. MEAN RADIAL VELOCITY PROFILES. TWO VERTICAL STRAIGHT LINES, FROM THE DISC TOWARD THE CORE, INDICATE RESPECTIVELY THE EKMAN LAYER THICKNESSES $(\pi \sqrt{v / \Omega} / d)$ FOR THE CONDITIONS $R a=10^{9}$ and $10^{8} . x^{*}=x / d$ REPRESENTS THE RELATIVE AXIAL POSITION BETWEEN DISCS.
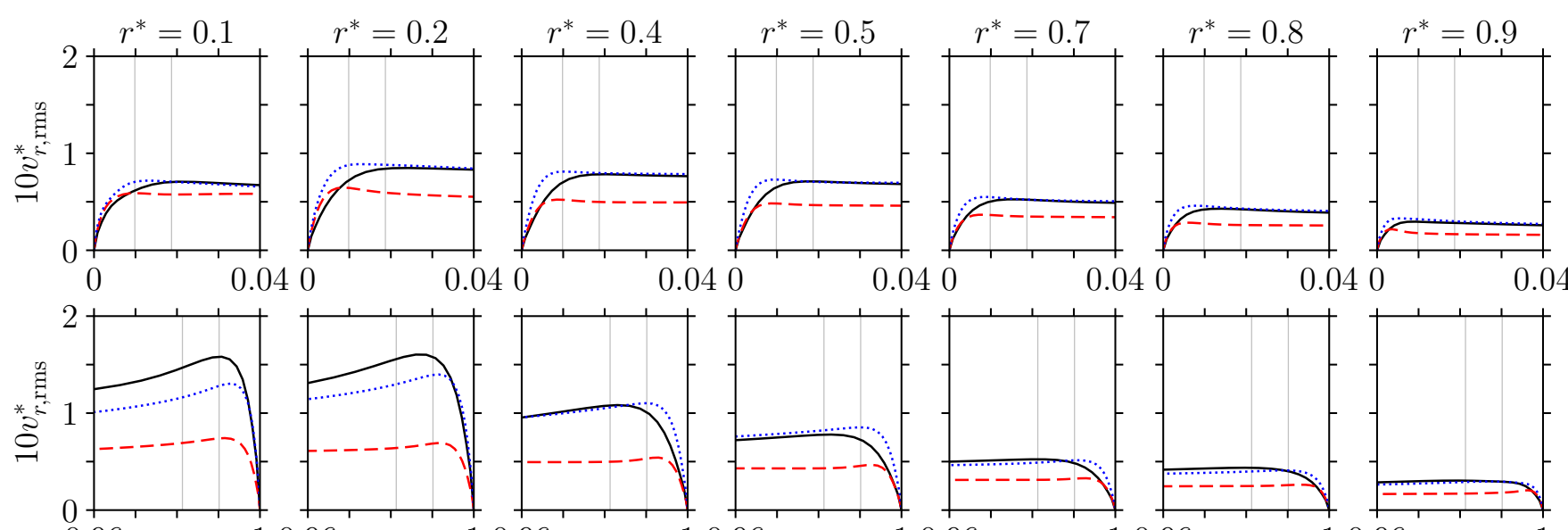

$$
0.96
$$

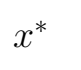

$10.96 \quad 10.96$

10.96

10.96

$-R a=10^{8}, B o=1.18 \quad--R a=10^{9}, B o=0.34$

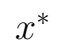

10.96

$x^{*}$

10.96

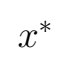

$x^{*}$

FIGURE 13. RADIAL VELOCITY FLUCTUATION PROFILES. TWO VERTICAL STRAIGHT LINES, FROM THE DISC TOWARD THE CORE, INDICATE RESPECTIVELY THE EKMAN LAYER THICKNESSES $(\pi \sqrt{v / \Omega} / d)$ FOR THE CONDITIONS $R a=10^{9}$ and $10^{8} . x^{*}=x / d$ REPRESENTS THE RELATIVE AXIAL POSITION BETWEEN DISCS.

This agrees with Lloyd and Moran's correlation for high $R a^{\prime}$ within $16 \%$ for the Rayleigh number range considered.

\subsection{Ekman Layer Scrubbing}

The analysis of the flow field has confirmed the unsteady Ekman layer behaviour for all the test cases considered in this paper. Velocity fluctuations (driving the Ekman layers) are large compared to mean radial velocities and are comparable to the velocity fluctuations in the sealed cavity. As discussed in section 4.2 for the sealed cavity, the Ekman layers may become turbulent at high rotational speeds and pressures, which would affect the disc heat transfer. Figure 19 shows levels of viscous dissipation are similar to those for the sealed cavity. However, in the presence of an axial throughflow, the core tangential velocity generates a mean radial outflow near the discs, and the internal energy may not accumulate at the Ekman layer. This is confirmed (for the present conditions) by the temperature contours in Fig. 15 which show no sign of hotter regions developing near the discs evident in Fig. 5 (c).

The present configuration is a relatively wide cavity with plane discs and an inner to outer radius ratio of $\sim 0.5$. For narrower cavities with cobs on the discs and lower radius ratios the 

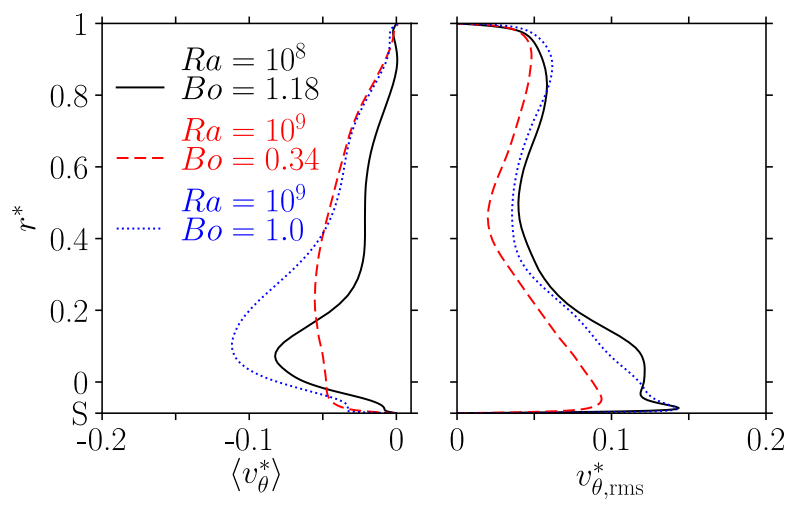

FIGURE 14. TANGENTIAL VELOCITY AND ITS FLUCTUATION PROFILES AT THE MID-AXIAL POSITION. "S" INDICATES SHAFT.
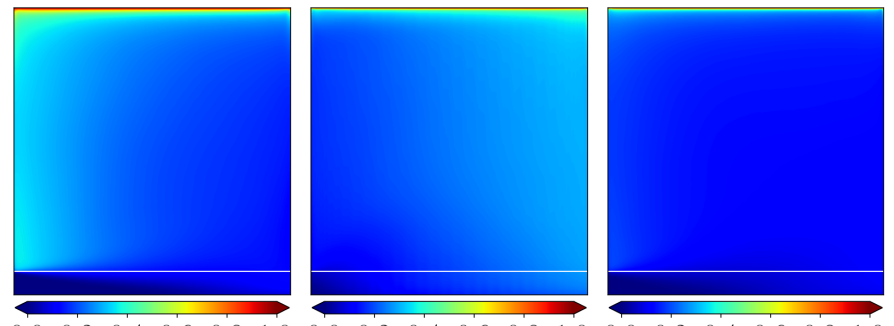

$\begin{array}{lllllll}0.0 & 0.2 & 0.4 & 0.6 & 0.8 & 1.0\end{array}$

(a) $R a=10^{8}, B o=1.18$

(b) $R a=10^{9}, B o=0.34$

(c) $R a=10^{9}, B o=1.0$

FIGURE 15. MEAN TEMPERATURE $\left\langle T^{*}\right\rangle$ CONTOUR. THE WHITE STRAIGHT LINE NEAR THE SHAFT CORRESPONDS TO THE DISC BORE RADIUS.

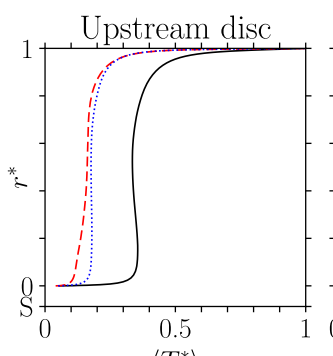

$\left\langle T^{*}\right\rangle$

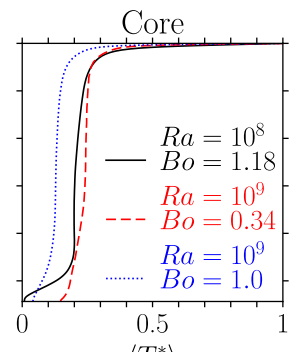

$\left\langle T^{*}\right\rangle$

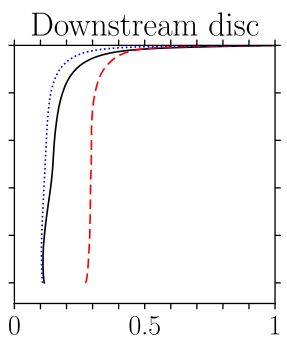

$\left\langle T^{*}\right\rangle$
FIGURE 16. MEAN TEMPERATURE PROFILES. "S" INDICATES SHAFT.

area available for interaction of the central throughflow with the main cavity flow will be lower, and so the effects of the axial throughflow may be reduced. In engine configurations, changes in inner disc radii (as shown in Fig. 1) may further complicate the influence of the throughflow. The present results confirm similarities of the shroud and outer disc flows for the sealed and open cavities but also indicate the need for further investigation and understanding of the interaction of throughflow and main cavity flows.

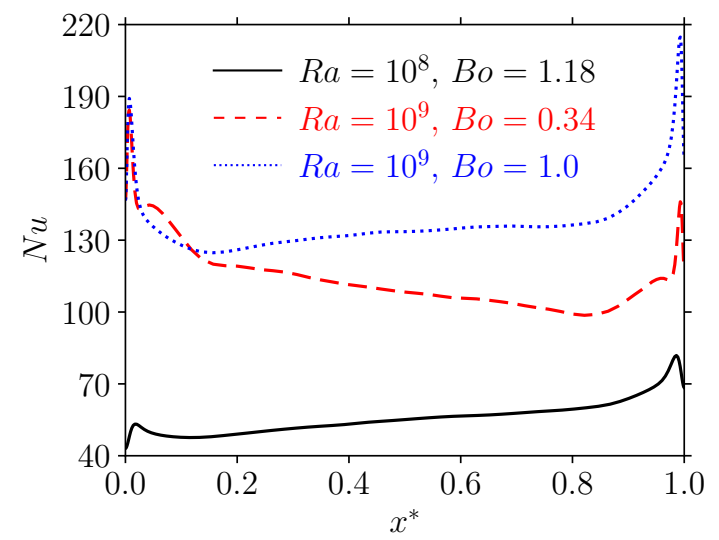

FIGURE 17. SHROUD NUSSELT NUMBER DISTRIBUTION.

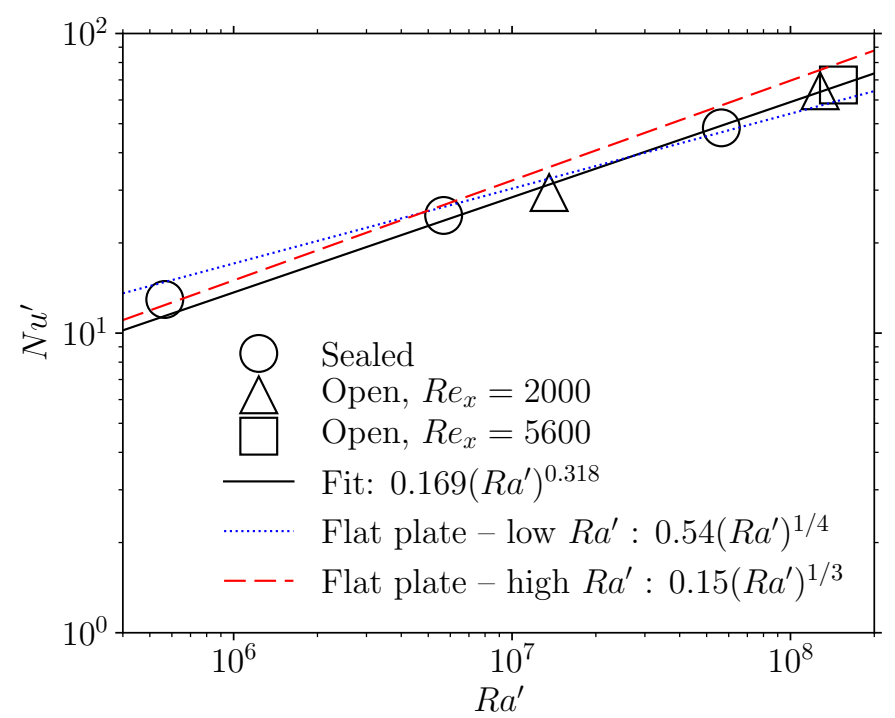

FIGURE 18. MODIFIED SHROUD NUSSELT NUMBER $N u^{\prime}$ AGAINST MODIFIED RAYLEIGH NUMBER $R a^{\prime}$.

\section{CONCLUSIONS}

LES and DNS studies of buoyancy-driven convection in sealed and open rotating cavities have been presented. Two different solvers have been compared for the sealed cavity configuration. The $R a$ and $R e_{x}$ considered are in the ranges $10^{7}-10^{9}$ and 2000-5600, respectively. The main conclusions are summarised as follows.

1. As shown in a previous study for a sealed rotating cavity [13], viscous heating effect due to Ekman layer scrubbing is mainly responsible for the difference in predicted shroud $\mathrm{Nu}$ by two solvers. In the Ekman layer velocity fluctuates around a near-zero mean, the internal energy converted from the viscous dissipation is unable to convect away but accumulates to heat up the disc. This effect is associated with the Eckert number. The viscous dissipation effect is not in- 

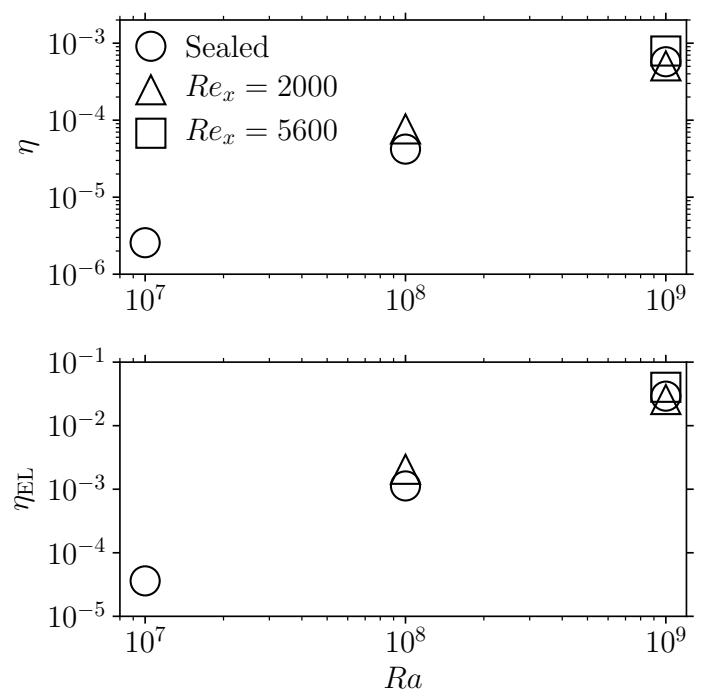

FIGURE 19. TOP: RAITO OF DISSIPATION TO TOTAL HEAT CONVECTION. BOTTOM: RATIO OF DISSIPATION PER UNIT VOLUME OF THE EKMAN LAYER TO HEAT CONVECTION PER UNIT VOLUME OF THE CAVITY.

cluded in the incompressible solver using the Boussinesq approximation.

2. Laminar Ekman layer behaviour on the discs is confirmed for all the conditions in this paper, except near the downstream disc bore affected by the axial throughflow jet impingement. The mean velocity is affected by $R a$ effects, whereas the velocity fluctuation is subject to $B o$ effects. Laminar Ekman layers are expected for the highest operating condition in Bohn et al.'s experiment. Transition to turbulent Ekman layers may occur at more extreme engine conditions, which can be evaluated using the equations given.

3. An equation is derived to scale the viscous heating due to Ekman layer scrubbing to engine conditions and shows excellent agreement with the LES data. The correlation indicates this effect is important at Bohn et al.'s highest speed condition. However, in the presence of an axial throughflow mean convection is likely to weaken this effect.

4. Large scale convective flow structures are confirmed for all the conditions studied with and without an axial throughflow.

5. The buoyancy number $B o$ appears to be a useful indicator to classify the buoyancy-driven convection with an axial throughflow. The two cases with a similar $B o$ shows similarities.

6. For the open cavity conditions considered in this paper the upstream disc temperature is subject to $R a$ effects, whilst the downstream disc temperature is more strongly influenced by Bo effects.

7. Using $N u^{\prime}$ and $R a^{\prime}$ based on the core-to-shroud temperature difference, the shroud heat transfer shows good agreement with a correlation for free convection between horizontal plates under gravity. Correlating the LES solutions gives

$$
N u^{\prime}=0.169\left(R a^{\prime}\right)^{0.318} \text {. }
$$

The present paper has built up confidence in using high fidelity DNS and LES approaches for predicting buoyancy-driven convection in rotating cavities. Future work will consider wall modelling to reduce computational costs for engine conditions.

\section{ACKNOWLEDGMENT}

The authors are grateful for the financial support provided by the Engineering and Physical Sciences Research Council under grant EP/P004091, and thank colleagues at the Universities of Surrey and Bath and Rolls-Royce plc for their technical support. The computing resources from ARCHER and local clusters in the University of Surrey are much appreciated.

\section{REFERENCES}

[1] Luberti, D., Tang, H., Scobie, J., Pountney, O., Owen, J., and Lock, G., 2019. "Influence of temperature distribution on radial growth of compressor discs". In Proceedings of ASME Turbo Expo 2019: Turbomachinery Technical Conference and Exposition. Paper No. GT2019-91848.

[2] Fitzpatrick, J. N., 2014. "Coupled thermal-fluid analysis with flowpath-cavity interaction in a gas turbine engine". Master thesis, Purdue University.

[3] Owen, J. M., and Long, C. A., 2015. "Review of buoyancyinduced flow in rotating cavities". Journal of Turbomachinery, 137(11), p. 111001.

[4] Atkins, N. R., 2013. "Investigation of a radial-inflow bleed as a potential for compressor clearance control". In Proceedings of ASME Turbo Expo 2013: Turbine Technical Conference and Exposition. Paper No. GT2013-95768.

[5] Sun, Z., Amirante, D., Chew, J. W., and Hills, N. J., 2016. "Coupled aerothermal modeling of a rotating cavity with radial inflow". Journal of Engineering for Gas Turbines and Power, 138(3), p. 032505.

[6] Bohn, D., Deuker, E., Emunds, R., and Gorzelitz, V., 1995. "Experimental and theoretical investigations of heat transfer in closed gas-filled rotating annuli”. Journal of Turbomachinery, 117(1), pp. 175-183.

[7] Bohn, D., and Gier, J., 1998. "The effect of turbulence on the heat transfer in closed gas-filled rotating annuli for different Rayleigh numbers". In Proceedings of ASME 1998 International Gas Turbine and Aeroengine Congress and Exhibition. Paper No. 98-GT542.

[8] Sun, Z., Kilfoil, A., Chew, J. W., and Hills, N. J., 2004. "Numerical simulation of natural convection in stationary and rotating cavities". In Proceedings of ASME Turbo Expo 2004: Power for Land, Sea, and Air. Paper No. GT2004-53528.

[9] King, M. P., Wilson, M., and Owen, J. M., 2007. "RayleighBénard convection in open and closed rotating cavities". Journal of Engineering for Gas Turbines and Power, 129(2), pp. 305-311.

[10] Pitz, D. B., Chew, J. W., Marxen, O., and Hills, N. J., 2017. "Direct numerical simulation of rotating cavity flows using a spectral element-Fourier method". Journal of Engineering for Gas Turbines and Power, 139(7), p. 072602. 
[11] Pitz, D. B., Chew, J. W., and Marxen, O., 2018. "Large-eddy simulation of buoyancy-induced flow in a sealed rotating cavity". Journal of Engineering for Gas Turbines and Power, 141(2), p. 021020 .

[12] Gao, F., Chew, J. W., and Pitz, D. B., 2019. "Numerical study of buoyancy-driven flow in a closed rotating annulus". In Proceedings of Global Power and Propulsion Society. Paper No. GPPSBJ-2019-0034.

[13] Gao, F., Pitz, D. B., and Chew, J. W., 2020. "Numerical investigation of buoyancy-induced flow in a sealed rapidly rotating disc cavity". International Journal of Heat and Mass Transfer, 147, p. 118860 .

[14] Tang, H., and Owen, J. M., 2017. "Theoretical model of buoyancyinduced heat transfer in closed compressor rotors". Journal of Engineering for Gas Turbines and Power, 140(3), p. 032605.

[15] Pitz, D. B., Chew, J. W., and Marxen, O., 2019. "Effect of an axial throughflow on buoyancy-induced flow in a rotating cavity". International Journal of Heat and Fluid Flow, 80, p. 108468.

[16] Hollands, K. G. T., Raithby, G. D., and Konicek, L., 1975. "Correlation equations for free convection heat transfer in horizontal layers of air and water". International Journal of Heat and Mass Transfer, 18(7), pp. 879-884.

[17] Grossmann, S., and Lohse, D., 2000. "Scaling in thermal convection: a unifying theory". Journal of Fluid Mechanics, 407, pp. 27-56.

[18] Farthing, P. R., Long, C. A., Owen, J. M., and Pincombe, J. R., 1992. "Rotating cavity with axial throughflow of cooling air: flow structure". Journal of Turbomachinery, 114(1), pp. 237-246.

[19] Farthing, P. R., Long, C. A., Owen, J. M., and Pincombe, J. R., 1992. "Rotating cavity with axial throughflow of cooling air: heat transfer". Journal of Turbomachinery, 114(1), pp. 229-236.

[20] Bohn, D. E., Deutsch, G. N., Simon, B., and Burkhardt, C., 2000. "Flow visualisation in a rotating cavity with axial throughflow". In Proceedings of ASME Turbo Expo 2000: Power for Land, Sea and Air. Paper No. 2000-GT-0280.

[21] Long, C. A., Alexiou, A., and Smout, P. D., 2003. "Heat transfer in H.P. compressor gas turbine internal air systems: measurements from the peripheral shroud of a rotating cavity with axial throughflow". In Proceedings of the 2nd International Conference on Heat Transfer, Fluid Mechanics, and Thermodynamics. Paper No. LC1.

[22] Long, C. A., and Childs, P. R. N., 2007. "Shroud heat transfer measurements inside a heated multiple rotating cavity with axial throughflow". International Journal of Heat and Fluid Flow, 28(6), pp. 1405-1417.

[23] Long, C. A., Miché, N. D. D., and Childs, P. R. N., 2007. "Flow measurements inside a heated multiple rotating cavity with axial throughflow". International Journal of Heat and Fluid Flow, 28(6), pp. 1391-1404.

[24] Atkins, N. R., and Kanjirakkad, V., 2014. "Flow in a rotating cavity with axial throughflow at engine representative conditions". In Proceedings of ASME Turbo Expo 2014: Turbine Technical Conference and Exposition. Paper No. GT2014-27174.

[25] Puttock-Brown, M. R., Rose, M. G., and Long, C. A., 2017. "Experimental and computational investigation of Rayleigh-Bénard flow in the rotating cavities of a core compressor". In Proceedings of ASME Turbo Expo 2017: Turbomachinery Technical Conference and Exposition. Paper No. GT2017-64884.

[26] Puttock-Brown, M. R., and Rose, M. G., 2018. "Formation and evolution of Rayleigh-Bénard streaks in rotating cavities". In Pro- ceedings of ASME Turbo Expo 2018: Turbine Technical Conference and Exposition. Paper No. GT2018-75497.

[27] Lloyd, J. R., and Moran, W. R., 1974. "Natural convection adjacent to horizontal surface of various planforms". Journal of Heat Transfer, 96(4), pp. 443-447.

[28] Bohn, D., Ren, J., and Tuemmers, C., 2006. "Investigation of the unstable flow structure in a rotating cavity". In Proceedings of ASME Turbo Expo 2006: Power for Land, Sea and Air. Paper No. GT2006-90494.

[29] Sun, Z., Lindblad, K., Chew, J. W., and Young, C., 2006. "LES and RANS investigations into buoyancy-affected convection in a rotating cavity with a central axial throughflow". Journal of Engineering for Gas Turbines and Power, 129(2), pp. 318-325.

[30] Tian, S., Tao, Z., Ding, S., and Xu, G., 2008. "Computation of buoyancy-induced flow in a heated rotating cavity with an axial throughflow of cooling air". International Journal of Heat and Mass Transfer, 51(3-4), pp. 960-968.

[31] Tan, Q., Ren, J., and Jiang, H., 2009. "Prediction of flow features in rotating cavities with axial throughflow by RANS and LES". In Proceedings of ASME Turbo Expo 2009: Power for Land, Sea and Air. Paper No. GT2009-59428.

[32] Tan, Q., Ren, J., and Jiang, H., 2014. "Prediction of 3d unsteady flow and heat transfer in rotating cavity by discontinuous Galerkin method and transition model". In Proceedings of ASME Turbo Expo 2014: Turbine Technical Conference and Exposition. Paper No. GT2014-26584.

[33] Amirante, D., and Hills, N. J., 2015. "Large-eddy simulations of wall bounded turbulent flows using unstructured linear reconstruction techniques". Journal of Turbomachinery, 137(5), p. 051006.

[34] Blackburn, H. M., and Sherwin, S. J., 2004. "Formulation of a Galerkin spectral element-Fourier method for three-dimensional incompressible flows in cylindrical geometries". Journal of Computational Physics, 197(2), pp. 759-778.

[35] Gao, F., Poujol, N., Chew, J. W., and Beard, P. F., 2018. "Advanced numerical simulation of turbine rim seal flows and consideration for RANS turbulence modelling". In Proceedings of ASME Turbo Expo 2018: Turbomachinery Technical Conference and Exposition. Paper No. GT2018-75116.

[36] El Khoury, G. K., Schlatter, P., Noorani, A., Fischer, P. F., Brethouwer, G., and Johansson, A. V., 2013. "Direct numerical simulation of turbulent pipe flow at moderately high Reynolds numbers". Flow, Turbulence and Combustion, 91(3), pp. 475495.

[37] Ahlers, G., Grossmann, S., and Lohse, D., 2009. "Heat transfer and large scale dynamics in turbulent Rayleigh-Bénard convection". Reviews of Modern Physics, 81, pp. 503-537.

[38] Greenspan, H. P., 1968. The theory of rotating fluids. Cambridge University Press.

[39] Owen, J. M., and Pincombe, J. R., 1980. "Velocity measurements inside a rotating cylindrical cavity with a radial outflow of fluid". Journal of Fluid Mechanics, 99(1), pp. 111-127.

[40] Kilfoil, A. S. R., and Chew, J. W., 2009. "Modelling of buoyancyaffected flow in co-rotating disc cavities". In Proceedings of ASME Turbo Expo 2009: Power for Land, Sea, and Air. Paper No. GT2009-59214.

[41] Owen, J. M., and Pincombe, J. R., 1979. "Vortex breakdown in a rotating cylindrical cavity". Journal of Fluid Mechanics, 90(1), pp. 109-127. 